\title{
Management of late endocardial lead dislocation into the pulmonary trunk
}

Diana Paskudzka1 ${ }^{1, A-F}$, Agnieszka Kołodzińska ${ }^{1, A-F}$, Przemysław Stolarz ${ }^{1, B-C, E-F}$, Łukasz Łyżwiński ${ }^{1, E-F}$, Marcin Grabowski $^{1, \mathrm{C}, \mathrm{E}-\mathrm{F}}$, Andrzej Kutarski ${ }^{2, \mathrm{C}, \mathrm{E}-\mathrm{F}}$, Grzegorz Opolski ${ }^{1, \mathrm{~A}, \mathrm{C}, \mathrm{E}-\mathrm{F}}$

A - Research concept and design, B - Collection and/or assembly of data, C - Data analysis and interpretation,

D - Writing the article, E - Critical revision of the article, F - Final approval of article

1. $1^{\text {st }}$ Department of Cardiology, Medical University of Warsaw

2. Department of Cardiology, Medical University, Lublin, Poland

Address for correspondence:

Diana Paskudzka, $1^{\text {st }}$ Department of Cardiology, Medical University of Warsaw

email: paskudzkadiana@gmail.com

Agnieszka Kołodzińska, $1^{\text {st }}$ Department of Cardiology, Medical University of Warsaw

email: aa.kolodzinska@wp.pl

Przemysław Stolarz, $1^{\text {st }}$ Department of Cardiology, Medical University of Warsaw email: pstolarz@wum.edu.pl

Łukasz Łyżwiński, $1^{\text {st }}$ Department of Cardiology, Medical University of Warsaw email: luke.lyzwinski@gmail.com

Marcin Grabowski, $1^{\text {st }}$ Department of Cardiology, Medical University of Warsaw email: marcin.grabowski@wum.edu.pl

Andrzej Kutarski, Department of Cardiology, Medical University, Lublin, Poland email: a_kutarski@yahoo.com

Grzegorz Opolski, $1^{\text {st }}$ Department of Cardiology, Medical University of Warsaw email: grzegorz..opolski@wum.edu.pl

Received: 2018-06-22

Revised: 2018-11-06

Accepted: 2018-11-12

Final review: 2018-11-12

DOI: $10.24255 / \mathrm{hbj} / 99705$

Key words:

lead removal, pacemaker-related complication, lead dislocation into the pulmonary trunk

\section{Abstract}

\section{Background}

Late migration of the endocardial lead into the pulmonary trunk or even as far as the pulmonary arteries is a rare complication of cardiac implantable electronic devices (CIED). There are no available guidelines or any general consensus on how to treat such cases.

\section{Material and methods}

8979 patients underwent implantation or replacement of a CIED at the Department of Cardiology of the Medical University of Warsaw. Most patients had a routine echocardiography (echo) examination before and both echo and chest X-ray after the procedure, as well as an echo examination during the follow-up. Chest X-ray was also performed during the follow-up visit when indicated. In some cases chest X-ray or computed tomography was also performed for non-cardiological reasons. In this patient population we identified 3 cases with a lead dislocation into the pulmonary trunk.

\section{Results}

Patient $A$ underwent conservative treatment, patient $B$ underwent a reduction of the lead body excess during the pulse 
generator replacement, and patient $C$ underwent transvenous lead removal and a new CIED was implanted. The follow-up revealed that patient $A$ presented a stable lead position in chest $\mathrm{X}$-ray and stable electrical and echo parameters. Patient B in echo and X-ray presented a proper lead position. Patient C presented systematically increasing tricuspid valve regurgita- tion and features of pulmonary hypertension, with a proper position of the implanted lead in control echocardiography.

\section{Conclusion}

Both conservative and invasive treatments were relatively safe. However, analyses of larger patient cohorts are needed to determine the recommended method.

\section{INTRODUCTION}

Migration of the proximal ending or the excessive lead loop into the pulmonary trunk or even into the pulmonary arteries is a rare complication of cardiac electronic device implantation. Unfortunately, there are no available guidelines or any general consensus for managing such cases. Dislocation of the endocardial lead tip into the pulmonary trunk with the loss of pacing usually presents as an acute complication and is always resolved with correction of the lead position with or without its removal or replacement. Late complications are associated with thromboembolic events, infective endocarditis, lead-related ventricular tachyarrhythmias, and pulmonary hypertension.(1-6) At our center we followed up three patients who suffered from lead dislocation into the pulmonary trunk and each patient underwent a different therapeutic strategy.

\section{METHODS}

8979 patients underwent cardiovascular electronic device implantation, removal or pulse generator replacement at the Department of Cardiology of the Medical University of Warsaw. Most patients had routine echocardiography during the qualification process for CIED (implantation or replacement), echo and chest $X$-ray during qualification for lead removal, echo and chest $X$-ray after the procedure (implantation, removal or replacement), and echo during the follow-up. X-ray was performed at follow-up if indicated (e.g. invalid electrical or echocardiographic parameters). In some cases chest X-ray or computed tomography was performed for non-cardiological reasons. Electrical check-up of the pacemaker was performed every year while implantable cardioverter-defibrillators were checked every six months. Endocardial lead dislocation into the pulmonary trunk was diagnosed through echo and chest $\mathrm{X}$-ray in three patients.

\section{RESULTS}

Patient A was a 63-year-old woman with paroxysmal atrial fibrillation and an atrioventricular pacemaker implanted 20 years ago. Her indications for implantation were episodes of near syncope and syncope in the course of tachycardia-bradycardia syndrome. The ventricular lead loop spontaneously dislodged into the pulmonary trunk and the diagnosis was established about 10 years after implantation (Fig. A, B). The

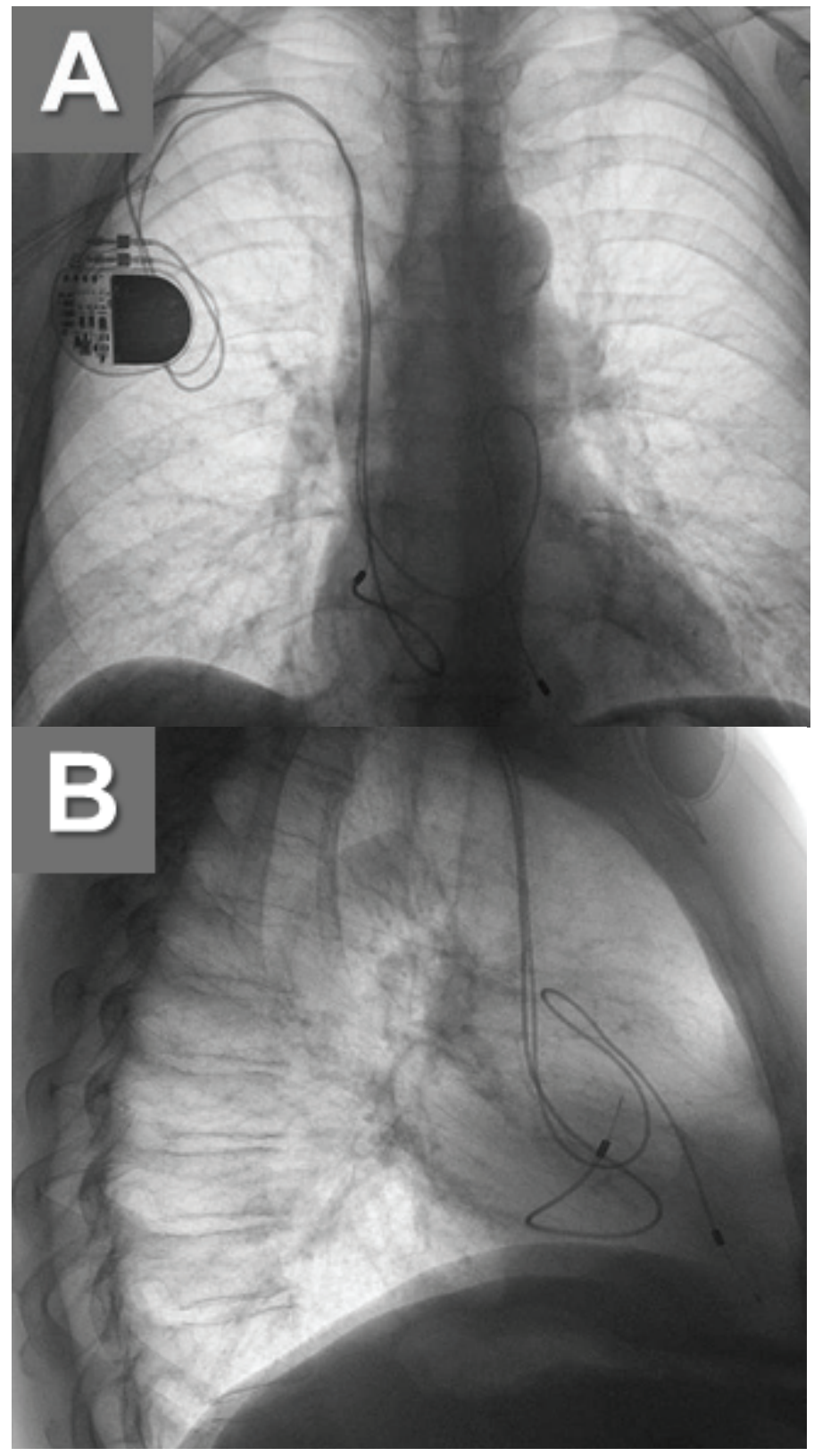

patient was on anticoagulant therapy, at first with vitamin $\mathrm{K}$ antagonist, and she is currently on a non-vitamin $\mathrm{K}$ antagonist. Transthoracic echocardiography revealed mild pulmonary artery and tricuspid valve regurgitation, without features 
of pulmonary hypertension, LVEF 59\%. During the device replacement it became apparent that the leads were stabilized by connective tissue, making it impossible to reposition them. The adopted strategy was to continue anticoagulant therapy and to repeat echocardiography every six months. As of the last follow-up (09.2018) the patient is in a stable condition.

Patient B was a 31-year-old woman with ventricular pacing. She was admitted to the clinic for planned pulse generator replacement due to end of the battery life. She had undergone implantation 10 years before due to sick sinus syndrome and atrioventricular conduction abnormalities with syncope. Due to anatomical complications and a systemic immunological condition she was qualified for single chamber pacing only. Her medical history includes thyroid storm in the course of Graves' disease, status post-allogenic bone marrow transplantation in 1984 due to congenital Diamond-Blackfan anemia, hepatitis B and hepatitis C. A chest radiograph made before the replacement showed a dislocation of the ventricular lead body into the pulmonary artery (Fig. C). The patient did not have any symptoms of pulmonary hypertension or pulmonary artery regurgitation and presented normal right ventricular
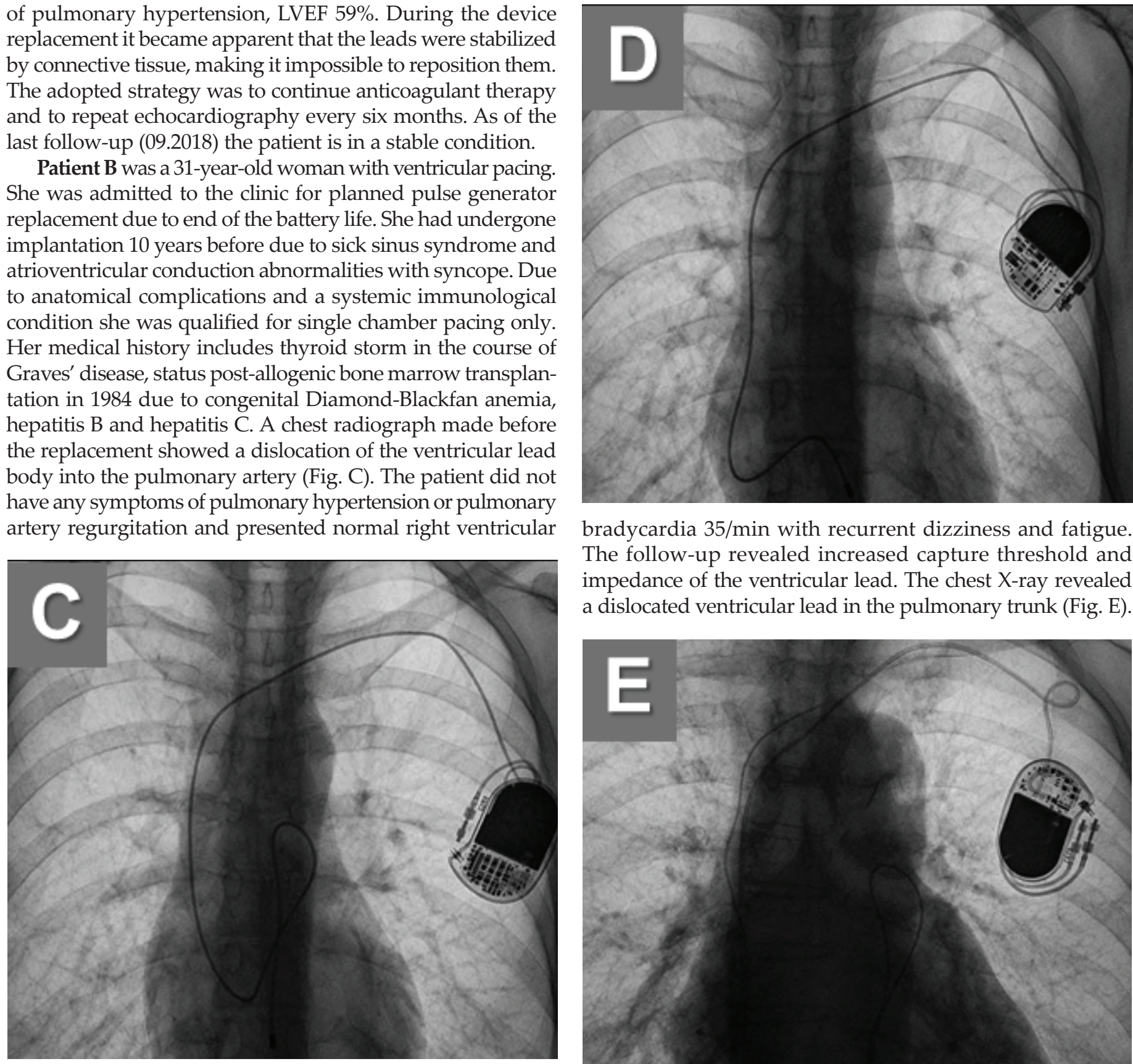

bradycardia 35/min with recurrent dizziness and fatigue. The follow-up revealed increased capture threshold and impedance of the ventricular lead. The chest X-ray revealed a dislocated ventricular lead in the pulmonary trunk (Fig. E).

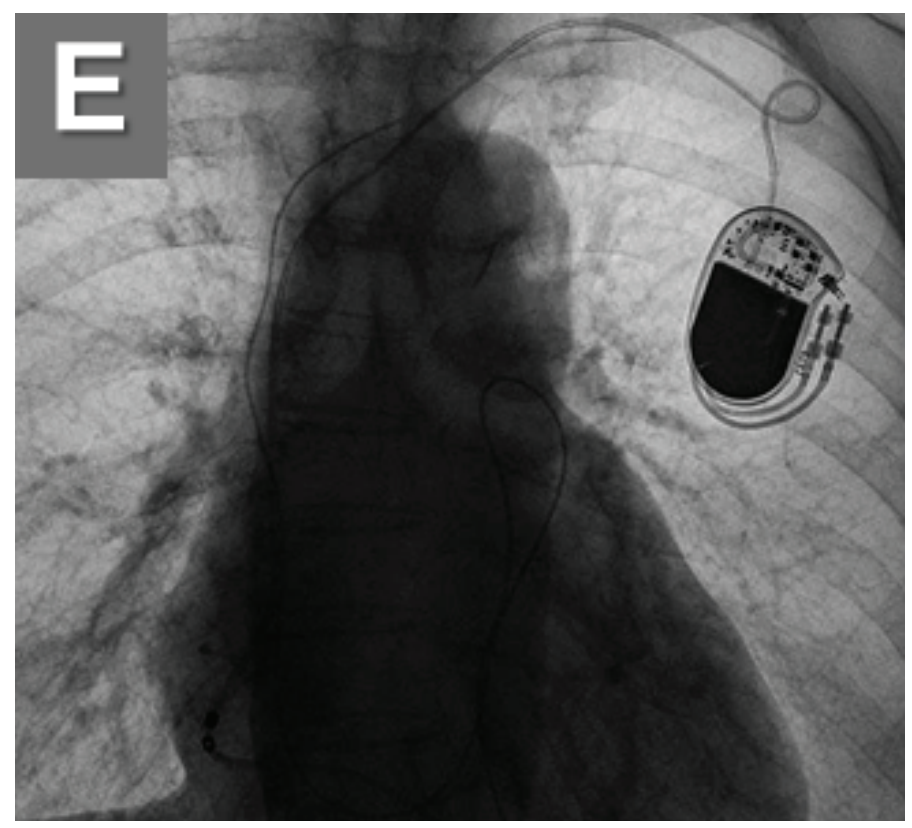

function in transthoracic echo. During the device replacement, lead repositioning and lead body excess reduction were successful. The chest $X$-ray confirmed the proper position of the lead in the right ventricle (Fig. D). Echocardiography showed moderate regurgitation of the tricuspid valve. Postoperative hospitalization was without complications. The most recent follow-up (07.2018) confirmed stable electrical and echocardiography parameters while chest $\mathrm{X}$-ray confirmed a proper lead position.

Patient $C$ was an 83-year-old, pacemaker-dependent woman. She was implanted with a VDD pacing system in 2002 due to a complete heart block. The lead was abandoned and a new VVI system was implanted in 2007 because of lead failure and also due to the end of the battery life. Two years later, she was admitted to the hospital due to symptomatic

In electrocardiography periodic ineffective ventricular pacing was observed. Echocardiographic examination revealed the ventricular lead in the pulmonary trunk and mild pulmonary valve regurgitation. The ventricular lead (dwelling time 89 months) was transvenously removed through the subclavian region while the VDD lead (dwelling time 171 months) was removed through femoral access using a pigtail and basket catheters. The procedure was clinically and radiologically successful. A new ventricular pacing system was implanted concomitantly. The VVI lead presented features of severe silicone insulation abrasion confirmed by optical microscope. 
Control examinations (echocardiography and chest X-ray) did not reveal any complication (Fig. F).

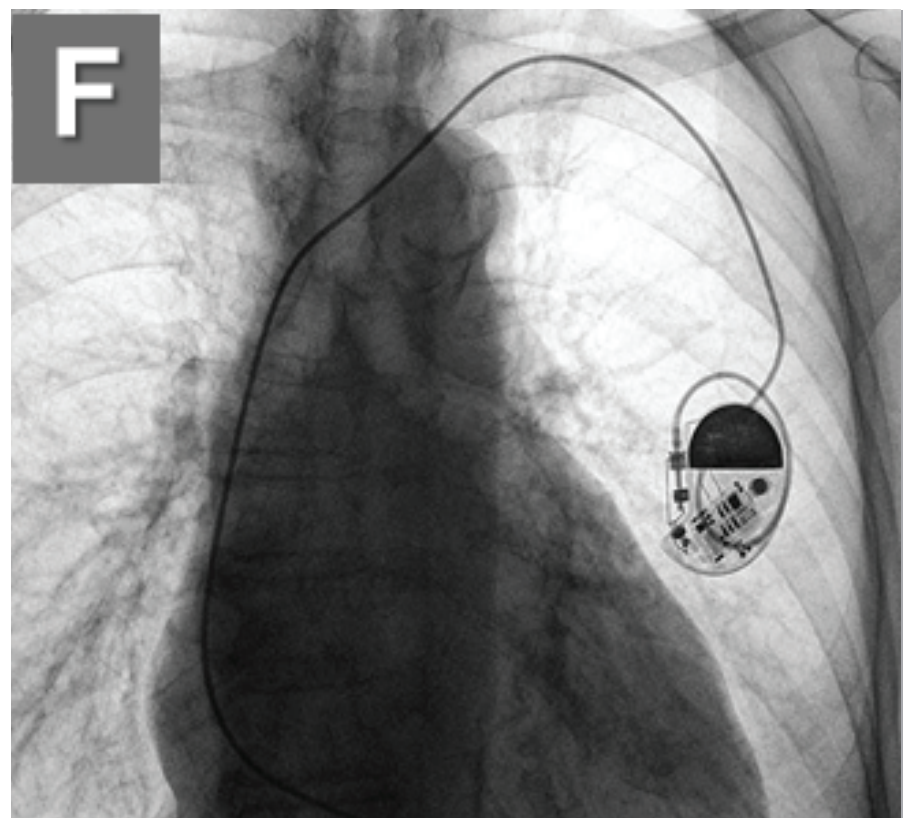

Table

\begin{tabular}{|l|l|l|l|l|l|l|l|}
\hline & DISLOCATION & MECHANISM & $\begin{array}{l}\text { MODE OF } \\
\text { TREATMENT }\end{array}$ & $\begin{array}{l}\text { CIED } \\
\text { implantation }\end{array}$ & $\begin{array}{l}\text { FOLLOW-UP } \\
\text { (since proce- } \\
\text { dure) }\end{array}$ & $\begin{array}{l}\text { ECHO } \\
\text { follow-up }\end{array}$ \\
\hline PATIENT A & $\begin{array}{l}\text { pulmonary } \\
\text { trunk }\end{array}$ & $\begin{array}{l}\text { pocket ligature } \\
\text { failure }\end{array}$ & conservative & DDD (1998) & $1998-2018$ & $-^{*}$ & $\begin{array}{l}\text { Stable lead } \\
\text { position }\end{array}$ \\
\hline PATIENT B & $\begin{array}{l}\text { pulmonary } \\
\text { trunk }\end{array}$ & $\begin{array}{l}\text { pocket ligature } \\
\text { failure }\end{array}$ & $\begin{array}{l}\text { reduction of } \\
\text { lead body } \\
\text { excess }\end{array}$ & VVI (2001) & $\begin{array}{l}2001-2018 \\
(2011-2018)\end{array}$ & $-^{*}$ \\
\hline PATIENT C & $\begin{array}{l}\text { pulmonary } \\
\text { trunk }\end{array}$ & $\begin{array}{l}\text { pocket ligature } \\
\text { failure }\end{array}$ & $\begin{array}{l}\text { transvenous } \\
\text { lead removal }\end{array}$ & $\begin{array}{l}\text { VDD (2002), } \\
\text { VVI (2007) }\end{array}$ & $\begin{array}{l}2002- \\
2013(2010- \\
2013)\end{array}$ & $\begin{array}{l}\text { TR 4/4; } \\
\text { pulmonary } \\
\text { hypertension }\end{array}$ & $\begin{array}{l}\text { Proper lead } \\
\text { position }\end{array}$ \\
\hline
\end{tabular}

*Without significant echocardiographic features of pulmonary hypertension and without pulmonary valve regurgitation.

\section{DISCUSSION}

Excessive lead loop dislocation into the pulmonary trunk due to pocket ligature failure was diagnosed in all three patients and each patient underwent a different treatment method. The approach strategies included: for patient A, conservative treatment with follow-ups and echocardiography every 6 months; for patient $B$, reduction of the excessive lead loop; for patient $C$, transvenous lead removal. The asymptomatic patients' observation was successfully conducted. (1-3; 5-6) Among 1767 patients who underwent transvenous lead removal, $19(9 \%)$ were diagnosed with a lead dislodged into the pulmonary bed. The proximal end was dislocated into the pulmonary artery in $47.4 \%$ and loop crossing the pulmonary valve was observed in $31.6 \%$ of the patients. The authors identified three different mechanisms of dislocation of the leads into the pulmonary trunk or the pulmonary arteries: lead fracture, excessive lead elongation due to pocket ligature failure, and non-functional lead dislodgement after cutting of the proximal ending. Removal was performed with a near $95 \%$ success rate, and the complication rate was similar to the group without lead dislocation. $(1,2)$ Kutarski et al. reported rare cases $(0.09 \%)$ of proximal lead end breakage during transvenous lead extraction (TLE), which was associated with dislodgment of the lead fragment into the pulmonary bed. The transvenous lead fragment retrieval did not increase the TLE-related procedure risk. The close observation of asymptomatic patients seems to be essential in cases with lead dislodgement into the pulmonary trunk. Transvenous lead removal in lead dislocations into the pulmonary trunk appears to be a relatively safe method.

\section{References}

1. Kutarski A, Polewczyk M, Pietura R. A rare complication of transvenous lead extraction - pulmonary embolization with a broken distal lead fragment. Heart Beat Journal. 2016;1:31-34. 
2. Polewczyk M, Jacheć W, Polewczyk AM, Polewczyk A, Czajkowski M, Kutarski A. Leads dislodged into the pulmonary vascular bed in patients with cardiac implantable electronic devices. Adv Interv Cardiol 2016; 12, 4 (46): 348-354.

3. Robinson T, Oliver J, Sheridan P, Sahu J, Bowes R. Fragmentation and embolization of pacemaker leads as a complication of lead extraction. Europace. 2010;12:754-5.

4. Michalak M, Kutarski A, Zawadzka-Byśko M, et al. Transvenous extraction of a broken atrial lead embolised into the pulmonary artery using a pigtail catheter. Kardiol Pol 2015; 73: 464.

5. Erkan H, Varol O, Karadeniz A et al. Embolisation of permanent pacemaker lead to pulmonary artery: a 15-year follow up. Kardiol Pol 2014; 72: 759.

6. Calvagna GM, Evola R, Valsecchi S. A complication of pacemaker lead extraction: pulmonary embolization of an electrode fragment. Europace. 2010;12:613. 